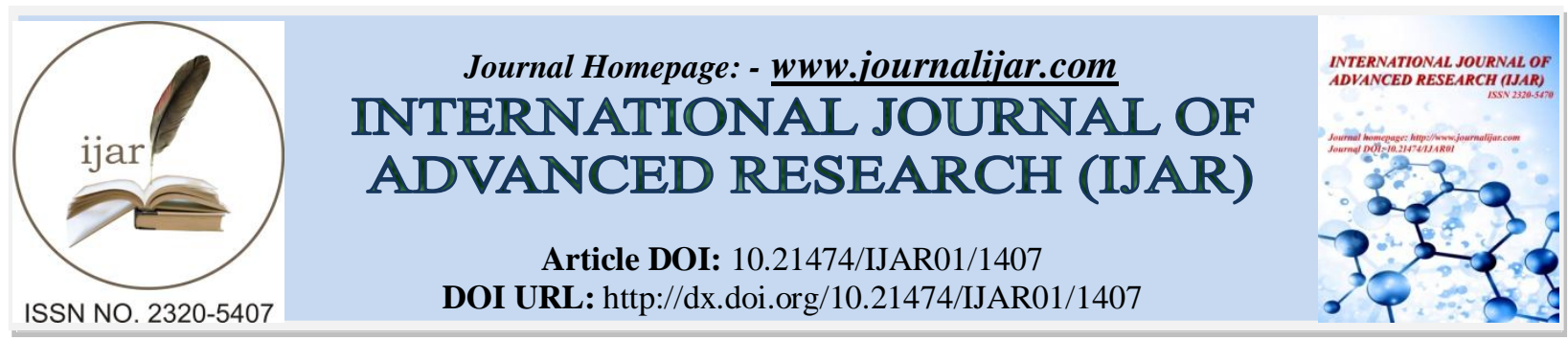

RESEARCH ARTICLE

\title{
OPTIMIZATION OF FORMULATION PARAMETERS OF EMTRICITABINE LOADED N- PALMITOYL GLUCOSAMINE NIOSOMES FOR BRAIN TARGETED DRUG DELIVERY
}

\author{
"Santha Sheela Natarajan Bhargavi ${ }^{1}$, Nappinnai Mohanavelu², Panneer Selvam Perumal ${ }^{3}$ and Sundhararajan \\ Ranganathan ${ }^{4}$. \\ 1. Research Scholar, C.L. Baid Metha College of Pharmacy, Jyothi Nagar, Thoraipakkam. \\ 2. Professor, Department of Pharmaceutics, Surya School of Pharmacy, Surya Group of Institutions, Vikravandi. \\ 3. Professor, Department of Pharmaceutical Chemistry, C.L.Baid Metha College of Pharmacy, Jyothi Nagar, \\ Thoraipakkam. \\ 4. Principal, Head of the Department, Department of Pharmaceutical Chemistry, Mohammed Sathak A.J. College \\ of Pharmacy, Medavakkam Road, Sholinganallur.
}

\section{Manuscript Info}

Manuscript History

Received: 12 June 2016

Final Accepted: 19 July 2016

Published: August 2016

Key words:-

Niosomes, Emtricitabine, Brain targeted drug delivery
Abstract

Emtricitabine is a nucleoside reverse transcriptase inhibitor (NRTI) used for the treatment of Human Immunodeficiency Virus (HIV) infection in adults and children. It is an intermediate central nervous system (CNS) penetrating drug with CNS Penetration-Effectiveness score of 0.5 . The HIV replication at sub therapeutic concentrations of the drug in the cerebrospinal fluid (CSF) leads to HIV associated neurocognitive disorders in AIDS patients. The use of glucose analogues as carriers for brain targeting has been widely investigated. Emtricitabine encapsulated niosomes were prepared by four different methods (thin layer evaporation (TLE)-vortex method, thin layer evaporation (TLE)-paddle stirring method, reverse phase evaporation method and proniosome method) with Span 40 and Span 60 as main surfactant. Cholesterol (CHL) and Solulan C24 (SOL) were also included in the niosomal formulations. Variations in compositions of the surfactant or surfactant-lipid mixture were made by varying the molar ratios of Span:CHL:SOL as 100:0:0 and 50:40:10. The total concentration of components ranged from 0.1 to $38 \mathrm{mM}$. The formulations were size reduced by sonication and size extrusion methods. The formulations were optimized for the method of preparation, concentrations and type of surfactant, total concentration of surfactant, hydration temperature and method of size reduction. The results showed that thin layer evaporation (TLE)-vortex method and thin layer evaporation (TLE)-paddle stirring method as efficient methods. The ratio of Span:CHL:SOL as 50:40:10, the total concentration of the surfactants to be $39 \mathrm{mM}$, sonication as the efficient method of size reduction and the hydration temperature as $65^{\circ} \mathrm{C}$. The optimized parameters were used for the preparation of Emtricitabine loaded N-palmitoyl glucosamine (NPG) niosomal preparations with Span 60 and Span 40 with the ratio of Span:CHL:SOL:NPG as 50:40:10:10. The niosomal suspensions were characterized for mean particle size, formation of vesicles, encapsulation efficiency (size 
exclusion chromatographic method and dialysis method) and evaluated for stability studies. The niosomal formulations were found to be stable at $4^{0} \mathrm{C}$ and $25^{\circ} \mathrm{C}$ for a period of 6 months. The formulation containing Span 60 was found to be the best formulation with good percentage entrapment.

Copy Right, IJAR, 2016,. All rights reserved.

\section{Introduction:-}

Niosomes or non-ionic surfactant vesicles or surfactant membrane vesicles are osmotically active and stable unilamellar or multilamellar vesicles wherein an aqueous solution is enclosed in highly ordered bilayer made up of non-ionic surfactant with or without cholesterol and dicetyl phosphate. They are vesicular systems similar to liposomes that can be used as carriers of amphiphilic and lipophilic drugs (Namdeo and Jain, 1996). Niosomes offer the advantages of having good stability, minimized toxicity, and minimized side effects and are also economically feasible with wider formulation versatility. The surfactants used for forming niosomes are biodegradable, nonimmunogenic and biocompatible. These vesicles improve the therapeutic efficacy of drugs by reducing the clearance rate, targeting to the specific site and by protecting the encapsulated drug. Drug targeting reduces the dose which leads to subsequent decrease in side effects (Brahmeshwar Mishra and Sarita Kumari Yadav; Madhav and Saini, 2001; Anchal Sankhyan and Pravin Pawar, 2012).

Glucose analogues as functionalized niosomes have been suggested as a tool to deliver drugs to the brain, considering the high level of cerebral glucose uptake. Thus, the derivatized surfactant N-palmitoyl glucosamine (NPG) has been successfully explored for targeting of drugs to brain. The recognition of glucosamine exposed on the surface of the vesicles by the glucose transporter GLUT-1 which is highly expressed on the BBB cells, has been hypothesized for the increased cerebral uptake (Macro Bragagni et al., 2012).

Emtricitabine is a nucleoside reverse transcriptase inhibitor (NRTI) used for the treatment of Human Immunodeficiency Virus (HIV) infection in adults and children. The cerebrospinal fluid (CSF) of HIV patients acts as a distinct virologic compartment for HIV-1 virus. In early stages, CSF and peripheral virus are similar but in later stages, CSF virus is phylogenetically distinct from virus in the periphery. The lower antiretroviral therapeutic concentrations of drug in CSF compared with that of plasma leads to persistent HIV replication with sub-therapeutic concentrations of Anti Retro Viral (ARV) drug in the nervous system and thus may subject the patients to the risk for HIV Associated Neurocognitive Disorders (HAND). ARV treatment regimens that can control CSF HIV replication may help to prevent HAND. Thus, penetration of drugs to the CNS and thereby increasing their concentration in the CSF plays a vital role in the effective treatment of Anti-Retroviral Therapy (ART) of HIV patients.

Emtricitabine is classified as a drug with intermediate CNS penetration with a CNS Penetration-Effectiveness (CPE) score of 0.5 (Peter Portegies and Mark van der Valk, 2010). The CSF/plasma ratio was about 36\% and that concentrations of emtricitabine exceed the wild-type $\mathrm{IC}_{50}$ in most individuals and may inhibit viral replication in the nervous system (Brookie M. Bestet et al., 2009).

By considering the potential benefits of functionalized niosomes as carriers to improve the delivery of drugs to brain, the use of Emtricitabine encapsulated glucose-bearing niosomal CNS targeted drug delivery may be a promising novel drug delivery system for increasing its concentrations in the brain to treat patients suffering with HIV Associated Neurocognitive Disorders.

\section{Materials And Methods:-}

Emtricitabine (Abhott Laboratories as gift sample); cholesterol (CHL), sorbitan monopalmitate (Span 40, HLB 9.8), sorbitan stearate (Span 60, HLB 4.7), sorbitol, sodium hydroxide and potassium dihydrogen ortho phosphate (SD Fine chemicals); Solulan C24 (Poly-24-oxyethylene cholesteryl ether, SOL), palmitic acid N-hydroxysuccinimide, glucosamine and Dulbecco's phosphate-buffered saline (DPBS) (Sigma-Aldrich) containing 2.7mM potassium chloride, $1.5 \mathrm{mM}$ potassium dihydrogen ortho phosphate, $137 \mathrm{mM}$ sodium chloride and $8.1 \mathrm{mM}$ disodium hydrogen orthophosphate. All other reagents used were of analytical grade. 


\section{Preparation Of Drug-Free Niosomes:-}

Niosomal suspensions were prepared by four different methods (thin layer evaporation (TLE)-vortex method, thin layer evaporation (TLE)-paddle stirring method, reverse phase evaporation method and proniosome method) with Span 40 and Span 60 as main surfactant. Cholesterol (CHL) and Solulan C24 (SOL) were also included in the niosomal formulations. Variations in compositions of the surfactant or surfactant-lipid mixture were made by varying the molar ratios of Span:CHL:SOL as 100:0:0 and 50:40:10. The total concentration of components ranged from 0.1 to $38 \mathrm{mM}$. The compositions of the various formulations are represented in table 1 .

The total concentration of components was fixed to $38 \mathrm{mM}$, since higher lipid amounts produces more viscous samples. The concentration of SOL was kept at $10 \mathrm{~mol} \%$, since high levels of SOL are potentially hemolytic (Uchegbu and Duncan, 1997).

\section{Method of Preparation:-}

\section{Thin layer evaporation (TLE)-vortex method:-}

The surfactant or surfactant-lipid mixture was dissolved in chloroform and introduced in a round-bottom flask. The solvent was evaporated under vacuum to form a thin layer on the flask wall. After hydration of the thin layer with 5 $\mathrm{mL}$ of phosphate buffer saline, 4 cycles of heating $\left(3 \mathrm{~min}\right.$ at $\left.65^{\circ} \mathrm{C}\right)$ and vortex mixing $(3 \mathrm{~min})$ were performed. The temperature of $65^{\circ} \mathrm{C}$ was selected since it is above the phase transition temperature of Span 60 , which is the component of the lipid mixture with the highest value of transition temperature $\left(50^{\circ} \mathrm{C}\right)$.

Thin layer evaporation (TLE)-paddle stirring method:-

The surfactant or surfactant-lipid thin layer obtained as above was hydrated with $25 \mathrm{~mL}$ of phosphate buffer saline. A paddle was then introduced in the flask and the suspension was stirred for $30 \mathrm{~min}$ at $65^{\circ} \mathrm{C}$.

\section{Reverse phase evaporation method:-}

Ten $\mathrm{mL}$ of the surfactant or surfactant-lipid mixture dissolved in chloroform was added to $5 \mathrm{~mL}$ of phosphate buffer saline. The resulted dispersion was sonicated for $3 \mathrm{~h}$ to form a W/O emulsion. Chloroform was then removed by rotary evaporation.

\section{Proniosome method:-}

Sorbitol was ground for $30 \mathrm{~min}$ at $24 \mathrm{~Hz}$ in a high-energy vibrational micromill. The 150-300 $\mu \mathrm{m}$ granulometric fractions were isolated and $1 \mathrm{~g}$ of this was added to a round-bottom flask connected to a rotary evaporator. The surfactant or surfactant-lipid mixture dissolved in chloroform $(3 \mathrm{~mL})$ was sequentially sprayed onto the powder surface by alternating with solvent evaporation. After addition of the last aliquot, the solvent was removed completely to obtain proniosomes. Hydration of proniosomes with $20 \mathrm{ml}$ of phosphate buffer saline resulted in niosomes (Macro Bragagni et al., 2012).

\section{Size Reduction Of Niosomes:-}

The formulated niosomal suspensions were centrifuged for $15 \mathrm{~min}$ at $4000 \mathrm{rpm}$. The supernatant was collected and divided into two portions of which one portion was subjected to sonication and the other to extrusion method for size reduction.

1. Sonication method: The niosomal suspensions were sonicated for 5 min with the instrument set at $60 \%$ of its maximum power.

2. Extrusion method: The niosomal suspensions were extruded 11 times through nitrocellulose membranes (Nucleopore $100 \mathrm{~nm}$, Whatman) using Extrusor Liposo Fast-Basic, Avestin, Inc.,Canada.

All the size reduced samples were stored at $4^{\circ} \mathrm{C}$ protected from the light (Macro Bragagni et al., 2012).

\section{Synthesis Of Glucose-Derivatized Surfactant N-Palmitoyl Glucosamine (NPG):-}

N-palmitoylglucosamine (NPG) was synthesized by slightly modified method of Dufes et al. Glucosamine (1.27 g) was added to a mixture of $1.5 \mathrm{~mL}$ of triethanolamine and $220 \mathrm{~mL}$ of DMSO. The mixture was stirred for $30 \mathrm{~min}$ at room temperature. Palmitic acid N-hydroxysuccinimide $(2.5 \mathrm{~g})$ dissolved in chloroform was then added to the mixture, left stirring for $72 \mathrm{~h}$ at room temperature, protected from the light. This was immersed in an ice bath and $100 \mathrm{~mL}$ of cold water was added. The precipitated product was collected by filtration, washed with water, DMSO and ethanol. Then it was dried at $40^{\circ} \mathrm{C}$ for $48 \mathrm{~h}$ and stored at $4^{\circ} \mathrm{C}$ protected from the light (Macro Bragagni et al., 2012). 


\section{Standard Calibration Of Emtricitabine In Distilled Water At 281 nm:-}

Accurately $100 \mathrm{mg}$ of the drug emtricitabine was weighed and dissolved in distilled water in a $100 \mathrm{~mL}$ standard volumetric flask. The volume was made up to the mark with distilled water. From this stock solution containing 1 $\mathrm{mg} / \mathrm{mL}$, solutions containing $5,10,15,20$ and $25 \mu \mathrm{g} / \mathrm{mL}$ concentrations of drug were prepared and the absorbencies determined spectrophotometrically at $281 \mathrm{~nm}$ using UV Visible Spectrophotometer. A graph was plot by taking concentration of drug on $\mathrm{X}$-axis and absorbance on $\mathrm{Y}$-axis and is represented in Figure 2 (Anindita Behera et al., 2011).

\section{Preparation Of NPG-Niosomes And Emtricitabine (FTC) Loaded-NPG-Niosomes:-}

Based on the results obtained on evaluation of the niosomal dispersions, the total concentration of components chosen for the preparation of NPG-niosomes and their composition by Span:CHL:SOL:NPG were optimized. After preparation, all suspensions were centrifuged and sonicated as described above.

The suitability of these techniques for drug-loaded vesicles preparation was assessed by determining the stability of emtricitabine under these experimental conditions by maintaining the drug solution containing $50 \mathrm{mg}$ of emtricitabine in $50 \mathrm{~mL}$ of distilled water under stirring for $1 \mathrm{~h}$ at $65^{\circ} \mathrm{C}$ and $90^{\circ} \mathrm{C}$, protected from the light. The residual unaltered drug was quantified by UV-visible spectrophotometric analysis at $281 \mathrm{~nm}$.

Drug (FTC)-loaded NPG-niosomes were prepared according to the optimized method, using the same concentrations and component ratios previously used for empty NPG-niosomes. The aqueous phase consisted of 20 $\mathrm{mL}$ emtricitabine solution $(2 \mathrm{mg} / \mathrm{mL}$ ) (Macro Bragagni et al., 2012).

\section{Characterization Of Niosomal Suspensions:-}

\section{Particle size of vesicles:-}

Particle size analysis was carried out using optical microscopy with calibrated eyepiece micrometer. About 200 niosomes were measured individually and the mean diameter was calculated. ${ }^{10}$ The results are represented in Fig 1 for drug free niosomes, Fig 3 for NPG-niosomes and Table 2 for FTC-NPG-niosomes.

\section{Formation of vesicles:-}

The effective formation of the vesicles was investigated by scanning electron microscopy (SEM). The SEM images are shown in Fig 4.

3. Drug encapsulation efficiency:-

Encapsulation efficiency of the niosomal dispersions were indirectly determined by using the following two methods:

\section{Size exclusion chromatography method:-}

From each sample of emtricitabine-NPG-niosomal dispersion, $0.5 \mathrm{~mL}$ of the dispersion was loaded onto a Sephadex G50 column (granulometry $20-80 \mathrm{~m}$ ) and eluted with water. Loaded-niosomes eluted as liquid, while free emtricitabine remained bound to the column. Vesicles were disrupted by addition of $0.05 \mathrm{~mL}$ TritonX-100 followed by ultra-centrifugation at $120000 \mathrm{~g}$ for $1 \mathrm{~h}$. The total drug amount in the niosomal dispersion and the drug amount actually encapsulated were determined by UV-visible spectrophotometric analysis at $281 \mathrm{~nm}$ in the supernatant after disrupting the vesicles before and after gel chromatography, respectively. The encapsulation efficiency was calculated according to the following equation:

$$
\mathrm{EE} \%=\frac{[\text { Encapsulated drug }] \times 100}{[\text { Total drug }]}
$$

All the data are the mean of five separate experiments. The results are tabulated in Table 2.

\section{Dialysis method:-}

From each of the emtricitabine-NPG-niosomal dispersion, $3 \mathrm{~mL}$ of the dispersion was dropped into a cellulose acetate dialysis bag (Spectra/Por ${ }^{\circledR}$, MW cut-off 12000), immersed in $150 \mathrm{~mL}$ of distilled water and magnetically stirred at $30 \mathrm{rpm}$. The unencapsulated drug was separated by replacing the receiving medium every $30 \mathrm{~min}$, until the level of dialyzed drug was undetectable. The collected samples after suitable dilutions were analyzed spectrophotometrically at $281 \mathrm{~nm}$ using UV-visible spectrophotometer from which the amount of drug dialyzed were determined. The total amount of drug initially present in each niosomal suspension was determined by disrupting the vesicles by addition of TritonX-100 followed by ultra-centrifugation (as described above) of $1 \mathrm{~mL}$ of non-dialyzed sample (to disrupt the vesicles) followed by emtricitabine assay in the supernatant by UV-visible 
spectophotometric analysis at $281 \mathrm{~nm}$. The percent of encapsulation efficiency (EE\%) was determined by the following equation:

$$
\mathrm{EE} \%=\frac{[\text { Total drug }]-[\text { Diffused drug }]}{[\text { Total drug }]} \text { X100 }
$$

Each result is the mean of three separate experiments. The results are tabulated in Table 2 (Macro Bragagni et al., 2012; Kandasamy Ruckmani and Veintramuthu Sankar, 2010).

\section{Stability Studies Of Niosomal Dispersions:-}

Stability studies of drug-loaded and unloaded niosomal dispersions were carried out by storing the niosomal suspensions protected from light and sealed at $4^{\circ} \mathrm{C}$ and $25^{\circ} \mathrm{C}$. The characterization parameters like particle size and encapsulation efficiency (determined after separation of loaded from un-loaded drug by size exclusion chromatography) were evaluated immediately after the preparation and periodically with 3 months interval for a period of six months. The results are tabulated in Table 3 (Macro Bragagni et al., 2012).

\section{Results And Discussion:-}

Formulations of drug free niosomes were prepared by four different methods (Thin layer evaporation-vortex, Thin layer evaporation-paddle, Reverse phase evaporation method and Proniosome method) using two surfactants, Span 60 and Span 40 with cholesterol and Solulan C24. Variations were made in the molar ratios and total concentration of lipid components.

The formulated niosomes were evaluated for mean particle size. Formulations prepared by proniosome method were found to have considerably larger sized vesicles $(8.25 \pm 1.30,5.94 \pm 2.92,6.28 \pm 2.52,10.43 \pm 1.99,9.22 \pm 1.23$ and $10.64 \pm 2.05 \mu \mathrm{m}$ for formulations F1A4, F1B4, F1C4, F2A4, F2B4 and F2C4 respectively) indicating the aggregation of vesicles.

Formulations prepared by reverse phase evaporation posed problems in ensuring complete removal of chloroform in the final niosomal dispersion. Moreover, the vesicle sizes of these formulations were greater than other niosomal formulations indicating aggregation of vesicles. The values were found to be $5.07 \pm 1.48,3.24 \pm 0.81,5.85 \pm 0.65$, $7.43 \pm 1.50,3.10 \pm 0.55$ and $8.09 \pm 1.41 \mu \mathrm{m}$ for formulations F1A3, F1B3, F1C3, F2A3, F2B3 and F2C3 respectively.

Among all the formulations niosomes prepared by TLE-vortex $(5.81 \pm 3.02,3.13 \pm 0.62,5.75 \pm 0.84,8.91 \pm 2.89$, $3.46 \pm 1.09$ and $5.87 \pm 1.13 \mu \mathrm{m}$ for formulations F1A1, F1B1, F1C1, F2A1, F2B1 and F2C1 respectively) and TLE-paddle method $(6.01 \pm 3.41,3.15 \pm 0.65,5.75 \pm 0.84,9.06 \pm 2.97,3.52 \pm 1.14$ and $7.015 \pm 1.45 \mu \mathrm{m}$ for formulations F1A2, F1B2, F1C2, F2A2, F2B2 and F2C2 respectively) produced narrow sized niosomal dispersions. Moreover, vesicles formulated with Span alone (F1A1, F1A2, F1A3, F1A4, F2A1, F2A2, F2A3 and F2A4) yielded comparatively larger niosomes than those with cholesterol and Solulan added to it (F1B1, F1B2, F1B3, F1B4, F1C1, F1C2, F1C3, F1C4, F2B1, F2B2, F2B3, F2B4, F2C1, F2C2, F2C3 and F2C4).

Among the two methods of size reduction employed, probe sonication was found to be more satisfactory in reducing the vesicle size by approximately two times than that of size extrusion method for all formulations.

The suitability of the drug at different hydration temperatures were evaluated by subjecting the drug solution to $65^{0}$ and $90^{\circ} \mathrm{C}$. The results showed that the drug is stable and can be subjected to such temperatures.

Based on the results of mean particle size, formulations containing Span 60 and Span 40 with a total molar concentration of 38mM in the molar ratio of Span:CHL:SOL:NPG as 50:40:10:10 prepared by TLE-vortex (F1B1 and F2B1) and TLE-paddle (F1B2 and F2B2) were selected as the optimized formulations. The NPG drug-free niosomes (NPG-F1B1, NPG-F1B2, NPG-F2B1 and NPG-F2B2) were prepared by varying the hydration temperatures of $65^{\circ}$ and $90^{\circ} \mathrm{C}$. The mean particle size of the formulations prepared at the hydration temperature of $90^{\circ} \mathrm{C}$ showed slightly higher values than those prepared at $65^{\circ} \mathrm{C}$. So the hydration temperature was optimized to $65^{0} \mathrm{C}$.

Emtricitabine loaded NPG niosomes were prepared with Span 60 (FTC-NPG-F1B2) and Span 40 (FTC-NPG-F2B2) with a total molar concentration of $38 \mathrm{mM}$ in the molar ratio of Span:CHL:SOL:NPG as 50:40:10:10 TLE-paddle 
method at $65^{\circ} \mathrm{C}$. The drug loaded niosomes were characterized for mean particle size and evaluated for encapsulation efficiency by size exclusion method and dialysis method.

The results of particle size of niosomes were greater after probe sonication at $60 \%$ of its maximum power and so probe sonication was carried out at $80 \%$ of its maximum power to reduce the particle size. The resultant particle size analysis showed that mean particle size of formulation containing Span 40 (FTC-NPG-F2B2) was considerably greater than Span 60 (FTC-NPG-F1B2) whereas drug encapsulation was found to be more for Span 60 (FTC-NPGF1B2) compared with formulation containing Span 40 (FTC-NPG-F2B2) by both size exclusion method (66.67\% and $27.78 \%$ respectively) and dialysis method (64.70\% and $22.25 \%$ respectively).

The optimized drug-free NPG niosomal formulations and drug loaded formulations (NPG-F1B2, NPG-F2B2, FTCNPG-F1B2 and FTC-NPG-F2B2) were subjected to stability studies for a period of 6 months at $4^{0} \mathrm{C}$ and $25^{\circ} \mathrm{C}$. The results of mean vesicle size and encapsulation efficiency showed that the niosomes were considerably stable at both the temperatures.

\section{Summary And Conclusion:-}

Formulations of drug free niosomes were prepared by four different methods (Thin layer evaporation-vortex, Thin layer evaporation-paddle, Reverse phase evaporation method and Proniosome method) using two surfactants, Span 60 and Span 40 with cholesterol and Solulan C24. Variations were made in the molar ratios and total concentration of lipid components.

Among all the formulations niosomes prepared by TLE-vortex and TLE-paddle methods produced narrow sized niosomal dispersions.

Moreover, vesicles formulated with Span alone yielded comparatively larger niosomes than those with cholesterol and Solulan added to it.

Among the two methods of size reduction employed, probe sonication was found to be more satisfactory in reducing the vesicle size by approximately two times than that of size extrusion method for all formulations.

The suitability of the drug at different hydration temperatures was evaluated by subjecting the drug solution to $65^{0}$ and $90^{\circ} \mathrm{C}$. The results showed that the drug is stable and can be subjected to such temperatures.

Based on the results of mean particle size, formulations containing Span 60 and Span 40 with a total molar concentration of 38mM in the molar ratio of Span:CHL:SOL:NPG as 50:40:10:10 prepared by TLE-vortex and TLE-paddle were selected as the optimized formulations.

The NPG drug-free niosomes were prepared by varying the hydration temperatures of $65^{\circ}$ and $90^{\circ} \mathrm{C}$ and the hydration temperature was optimized to $65^{\circ} \mathrm{C}$.

Emtricitabine loaded NPG niosomes were prepared with Span 60 (FTC-NPG-F1B2) and Span 40 (FTC-NPG-F2B2) with a total molar concentration of $38 \mathrm{mM}$ in the molar ratio of Span:CHL:SOL:NPG as 50:40:10:10 by TLE-paddle method at $65^{\circ} \mathrm{C}$. The drug loaded niosomes were characterized for mean particle size and evaluated for encapsulation efficiency by size exclusion method and dialysis method.

The results of particle size of niosomes were greater after probe sonication at $60 \%$ of its maximum power and so probe sonication was carried out at $80 \%$ of its maximum power to reduce the particle size. The resultant particle size analysis showed that mean particle size of formulation containing Span 40 (FTC-NPG-F2B2) was considerably greater than Span 60 (FTC-NPG-F1B2) whereas drug encapsulation was found to be more for Span 60 (FTC-NPGF1B2) compared with formulation containing Span 40 (FTC-NPG-F2B2) by both size exclusion method (66.67\% and $27.78 \%$ respectively) and dialysis method (64.70\% and $22.25 \%$ respectively).

The optimized drug-free NPG niosomal formulations and drug loaded formulations (NPG-F1B2, NPG-F2B2, FTCNPG-F1B2 and FTC-NPG-F2B2) were subjected to stability studies for a period of 6 months at $4{ }^{\circ} \mathrm{C}$ and $25^{\circ} \mathrm{C}$. The results of mean vesicle size and entrapment efficiency showed that the niosomes were considerably stable at both the temperatures. 
To conclude, by considering the potential benefits of the drug formulated as niosomal dispersion, the optimized emtricitabine loaded NPG niosomal formulation (FTC-NPG-F1B2) which contains Span 60:CHL:SOL:NPG in the ratio of 50:40:10:10 with a total lipid concentration of $38 \mathrm{mM}$ and prepared by thin film hydration-paddle method may be considered as a potential alternative to improve brain targeting of emtricitabine and thus minimize HIV Associated Neurocognitive Disorders.

Table 1:- Formulation Of Drug-Free Niosomes ${ }^{5}$

\begin{tabular}{|c|c|c|c|c|}
\hline $\begin{array}{c}\text { FORMULATION } \\
\text { CODE }\end{array}$ & $\begin{array}{c}\text { METHOD OF } \\
\text { PREPARATION }\end{array}$ & COMPOSITION & MOL\% & $\begin{array}{c}\text { TOT.CONC. } \\
(\mathrm{mM})\end{array}$ \\
\hline \multicolumn{5}{|c|}{ Formulations containing Span 60} \\
\hline F1A1 & (TLE) - Vortex & \multirow{4}{*}{ Span 60} & \multirow{4}{*}{100} & \multirow{4}{*}{0.1} \\
\hline F1A2 & (TLE) - Paddle & & & \\
\hline F1A3 & RPEM & & & \\
\hline F1A4 & Proniosome & & & \\
\hline F1B1 & (TLE) - Vortex & \multirow{4}{*}{ Span 60:CHL:SOL } & \multirow{4}{*}{$50: 40: 10$} & \multirow{4}{*}{38} \\
\hline F1B2 & (TLE) - Paddle & & & \\
\hline F1B3 & RPEM & & & \\
\hline F1B4 & Proniosome & & & \\
\hline F1C1 & (TLE) - Vortex & \multirow{4}{*}{ Span 60:CHL:SOL } & \multirow{4}{*}{$50: 40: 10$} & \multirow{4}{*}{9.5} \\
\hline F1C2 & (TLE) - Paddle & & & \\
\hline F1C3 & RPEM & & & \\
\hline F1C4 & Proniosome & & & \\
\hline \multicolumn{5}{|c|}{ Formulations containing Span 40} \\
\hline F2A1 & (TLE) - Vortex & \multirow{4}{*}{ Span 40} & \multirow{4}{*}{100} & \multirow{4}{*}{0.1} \\
\hline F2A2 & (TLE) - Paddle & & & \\
\hline F2A3 & RPEM & & & \\
\hline F2A4 & Proniosome & & & \\
\hline F2B1 & (TLE) - Vortex & \multirow{4}{*}{ Span 40:CHL:SOL } & \multirow{4}{*}{$50: 40: 10$} & \multirow{4}{*}{38} \\
\hline F2B2 & (TLE) - Paddle & & & \\
\hline F2B3 & RPEM & & & \\
\hline F2B4 & Proniosome & & & \\
\hline F2C1 & (TLE) - Vortex & \multirow[t]{4}{*}{ Span 40:CHL:SOL } & \multirow[t]{4}{*}{$50: 40: 10$} & \multirow[t]{4}{*}{9.5} \\
\hline $\mathrm{F} 2 \mathrm{C} 2$ & (TLE) - Paddle & & & \\
\hline F2C3 & RPEM & & & \\
\hline F2C4 & Proniosome & & & \\
\hline
\end{tabular}

(TLE) - Vortex: Thin layer evaporation-vortex method

(TLE) - Paddle: Thin layer evaporation-paddle method

RPEM: Reverse phase evaporation method

CHL: Cholesterol

SOL: Solulan C24

TOT. CONC: Total concentration of the surfactant or surfactant-lipid mixture.

mM: Millimolar

MOL\%: Mole percent 
Fig 1:- Particle Size Of Drug-Free Niosomes

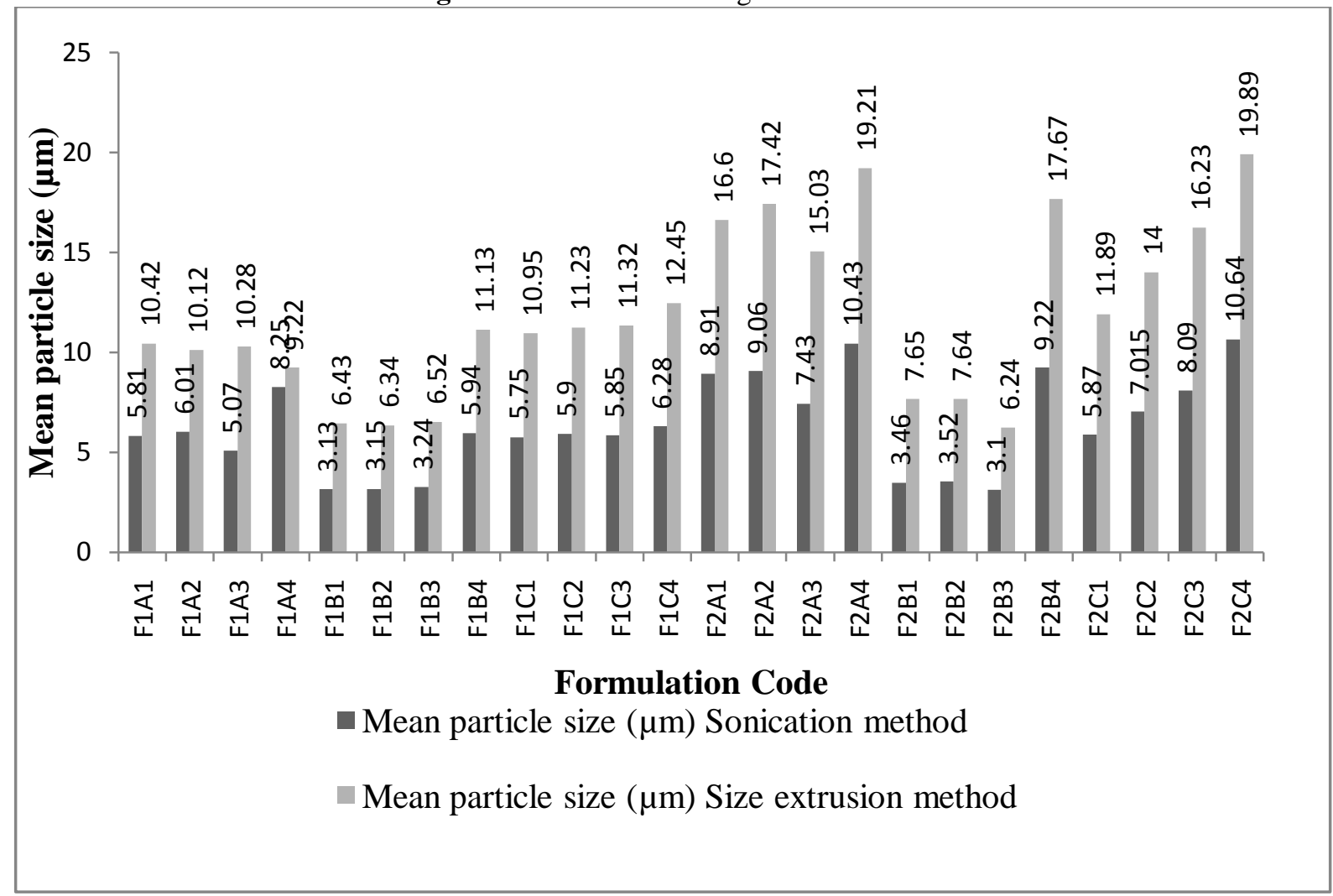

Fig 2:- Standard Calibration Graph Of FTC In Distilled Water At 281nm

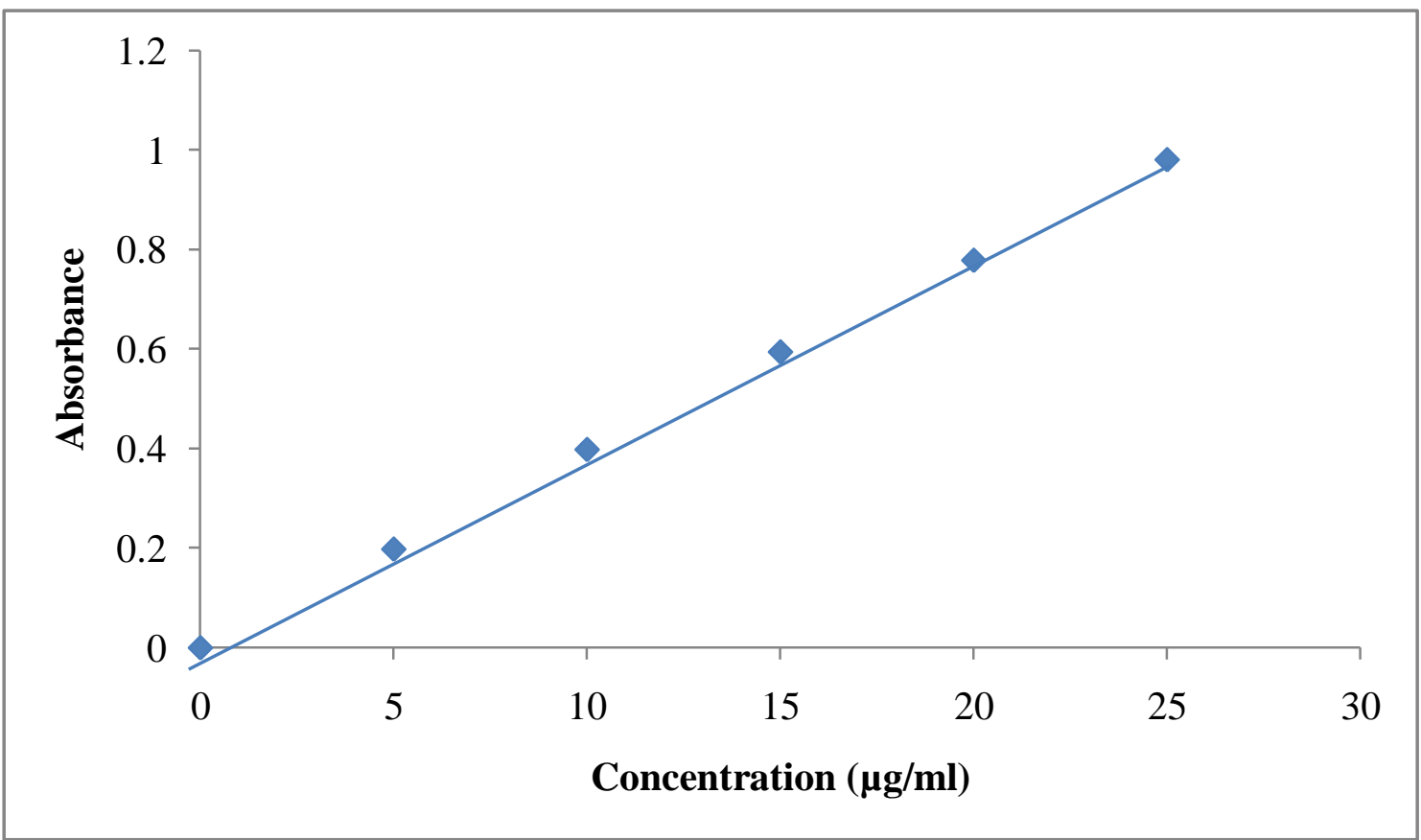


Fig 3:- Particle Size Of Drug-Free NPG Niosomes Prepared At Different Temperatures

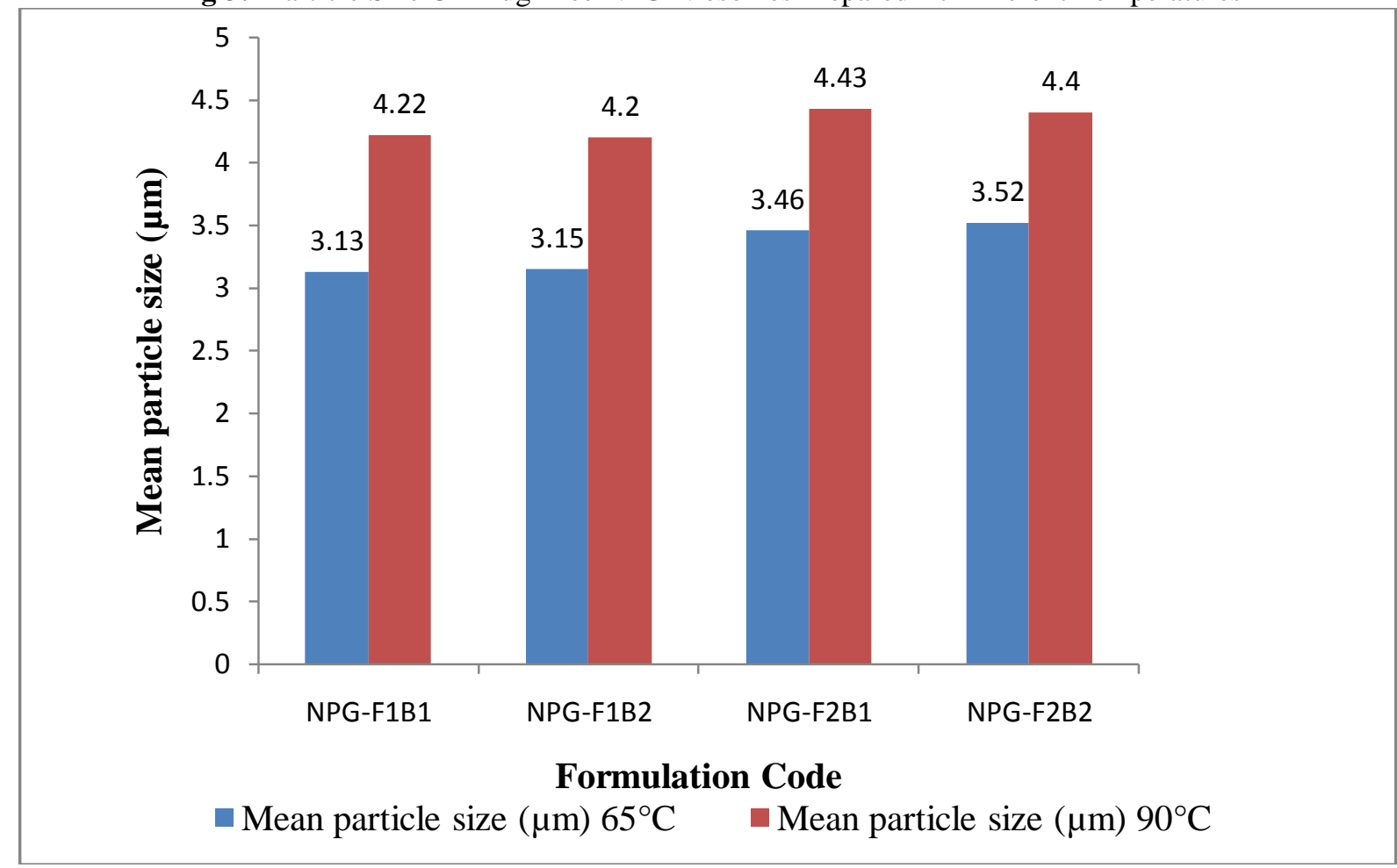

Fig 4:- Scanning Electron Microscopic Images Of Formulations Prepared By TLE-Paddle Method With A) SPAN 60

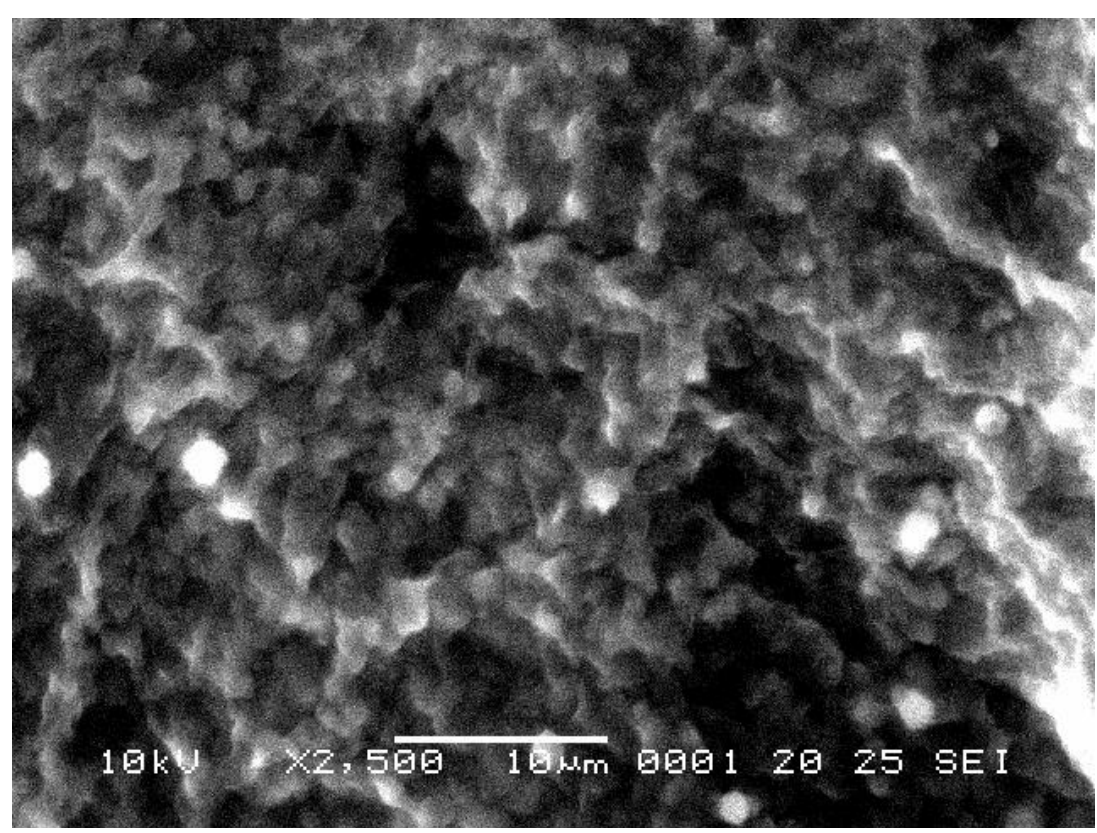




\section{B) SPAN 40}

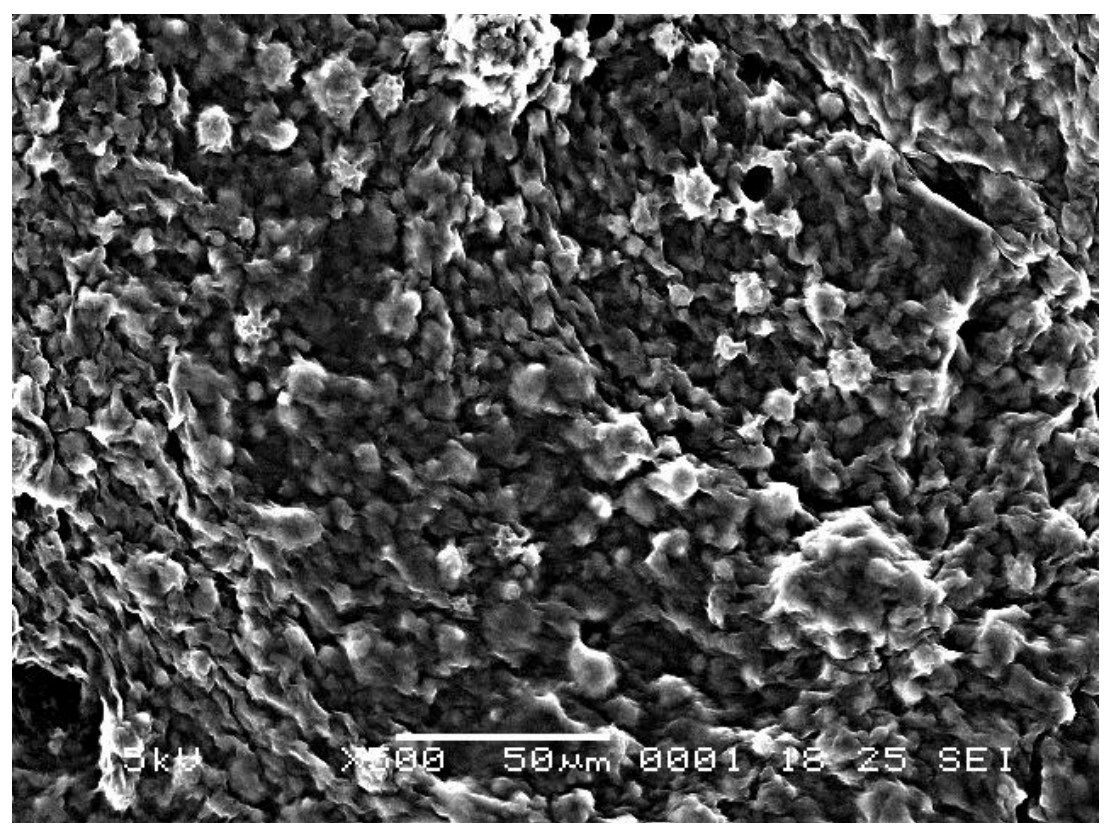

Table 2:- Particle Size And Encapsulation Efficiency Of FTC-NPG-Niosomes

\begin{tabular}{|l|l|l|l|l|}
\hline S.No. & Formulation code & $\begin{array}{l}\text { Mean particle size } \pm \text { S.D by } \\
\text { Sonication method }(\mathbf{n m})\end{array}$ & \multicolumn{2}{|c|}{\begin{tabular}{c} 
Encapsulation efficiency \pm SD (\%) \\
\cline { 3 - 4 }
\end{tabular}} \\
& & & $\begin{array}{c}\text { Size exclusion } \\
\text { chromatographic method }\end{array}$ & Dialysis method \\
\hline 1 & FTC-NPG-F1B2 & $154 \pm 4$ & $66.26 \pm 1.32$ & $64.45 \pm 1.14$ \\
\hline 2 & FTC-NPG-F2B2 & $169 \pm 4$ & $27.37 \pm 0.71$ & $22.46 \pm 0.91$ \\
\hline
\end{tabular}

Table 3:- Stability Of NPG-Niosomal Dispersion And FTC-NPG-Niosomal Dispersion Of Formulation F1B2

\begin{tabular}{|l|l|l|l|l|l|l|}
\hline S.No. & $\begin{array}{l}\text { Formulation } \\
\text { code }\end{array}$ & $\begin{array}{l}\text { Temperature } \\
\left({ }^{0} \mathbf{C}\right)\end{array}$ & Parameter & $\mathbf{0}$ day & $\mathbf{3}$ months & $\mathbf{6}$ months \\
\hline 1 & NPG-F1B2 & 4 & $\begin{array}{l}\text { Mean particle size } \\
\pm \text { SD }\end{array}$ & $157 \pm 4$ & $158 \pm 4$ & $158 \pm 4$ \\
\hline 2 & NPG-F1B2 & 25 & $\begin{array}{l}\text { Mean particle size } \\
\pm \text { SD }\end{array}$ & $157 \pm 4$ & $157 \pm 4$ & $158 \pm 4$ \\
\hline 3 & FTC-NPG-F1B2 & 4 & $\begin{array}{l}\text { Mean particle size } \\
\pm \text { SD }\end{array}$ & $153 \pm 3$ & $153 \pm 3$ & $155 \pm 3$ \\
\cline { 3 - 7 } & $\begin{array}{l}\text { Encapsulation } \\
\text { efficiency }\end{array}$ & $64.45 \pm 1.14$ & $64.93 \pm 1.83$ & $64.52 \pm 0.58$ \\
\hline 4 & FTC-NPG-F1B2 & 25 & $\begin{array}{l}\text { Mean particle size } \\
\pm \text { SD }\end{array}$ & $153 \pm 3$ & $154 \pm 3$ & $154 \pm 3$ \\
\cline { 3 - 6 } & & $\begin{array}{l}\text { Encapsulation } \\
\text { efficiency }\end{array}$ & $22.46 \pm 0.91$ & $22.61 \pm 0.75$ & $22.28 \pm 0.87$ \\
\hline
\end{tabular}

\section{References:-}

1. Anchal Sankhyan and Pravin Pawar. (2012): Recent trends in niosome as vesicular drug delivery system. Journal of Applied Pharmaceutical Science., 02(06): 20-32.

2. Anindita Behera., Aurobinda Parida., Amit Kumar Meher., Dannana Gowri Sankar., Swapan Kumar Moitra and Sudam Chandra Si. (2011): Development and validation of spectrophotometric method for determination of Emtricitabine and tenofovir disoproxil fumarate in bulk and tablet dosage form. Int. J. PharmTech Res., 3(3): 1874-1882.

3. Brahmeshwar Mishra and Sarita Kumari Yadav. Novel approaches for brain targeted drug delivery. In: Udupa $\mathrm{N}$ and Srinivas Mutalik. Recent trends in novel drug delivery. 337-370. 
4. Brookie M. Best., Scott Letendre., Edmund, V. Capparelli., Ronald Ellis., Steven S. Rossi., Peter Koopmans and Ignor Grant. (2009): CHARTER group. Efavirenz and emtricitabine concentrations consistently exceed wild-type $\mathrm{IC}_{50}$ in cerebrospinal fluid: CHARTER findings. Poster presentation. $16^{\text {th }}$ Conference on retroviruses and opportunistic infections. Feb 8-11, 2009, Montreal, Canada.

5. Kandasamy Ruckmani and Veintramuthu Sankar. (2010): Formulation and optimization of zidovudine niosomes. AAPS PharmSciTech., 11(3): 1119-1127.

6. Macro Bragagni., Natascia Mennini., Carla Ghelardini and Paola Mura. (2012): Development and characterization of niosomal formulations of doxorubicin aimed at brain targeting. J. Pharm. Pharmaceut. Sci., 15(1): 184-196.

7. Madhav, N.V.S. and Saini, A. (2001): Niosomes: A novel drug delivery system. International Journal of Research in Pharmacy and Chemistry., 1(3): 498-511.

8. Namdeo, A. and Jain, N.K. (1996): Niosomes as drug carriers. Indian Journal of Pharmaceutical Sciences., 58(2): 41-46.

9. Peter Portegies and Mark van der Valk. (2010): HIV associated CNS disease in the era of HAART - CSF/CNS penetration and efficacy. Presentation at the $5^{\text {th }}$ INTEREST workshop - $10-13$ May 2010, Dar-es-Salaam, Tanzania.

10. Uchegbu, I.F and Duncan, R. (1997): Niosomes containing N-(2-hydroxypropyl) methacrylamide copolymerdoxorubicin (PK1): effect of method of preparation and choice of surfactant on niosome characteristics and a preliminary study of body distribution. Int. J. Pharm., 155: 7-17. 